\title{
A comparative analysis of insertional effects in genetically engineered plants: considerations for pre-market assessments
}

\author{
Jaimie Schnell • Marina Steele · Jordan Bean • Margaret Neuspiel • \\ Cécile Girard • Nataliya Dormann • Cindy Pearson • Annie Savoie • \\ Luc Bourbonnière $\cdot$ Philip Macdonald
}

Received: 22 May 2014/Accepted: 16 October 2014/Published online: 26 October 2014

(C) The Author(s) 2014. This article is published with open access at Springerlink.com

\begin{abstract}
During genetic engineering, DNA is inserted into a plant's genome, and such insertions are often accompanied by the insertion of additional DNA, deletions and/or rearrangements. These genetic changes are collectively known as insertional effects, and they have the potential to give rise to unintended traits in plants. In addition, there are many other genetic changes that occur in plants both spontaneously and as a result of conventional breeding practices. Genetic changes similar to insertional effects occur in plants, namely as a result of the movement of transposable elements, the repair of
\end{abstract}

J. Schnell · C. Girard · P. Macdonald ( $\square)$

Plant and Biotechnology Risk Assessment Unit, Canadian

Food Inspection Agency, 1400 Merivale Road, Ottawa,

ON K1A 0Y9, Canada

e-mail: philip.macdonald@inspection.gc.ca

C. Girard

e-mail: cecile.girard@inspection.gc.ca

M. Steele · A. Savoie

Animal Feed Division, Canadian Food Inspection

Agency, 59 Camelot Drive, Ottawa, ON K1A 0Y9,

Canada

e-mail: marina.steele@inspection.gc.ca

A. Savoie

e-mail: annie.savoie@inspection.gc.ca

J. Bean · L. Bourbonnière

Food Directorate, Health Canada, 251 Sir Frederick

Banting Driveway, Ottawa, ON K1A 0K9, Canada

e-mail: jordan.bean@hc-sc.gc.ca double-strand breaks by non-homologous end-joining, and the intracellular transfer of organelle DNA. Based on this similarity, insertional effects should present a similar level of risk as these other genetic changes in plants, and it is within the context of these genetic changes that insertional effects must be considered. Increased familiarity with genetic engineering techniques and advances in molecular analysis techniques have provided us with a greater understanding of the nature and impact of genetic changes in plants, and this can be used to refine pre-market assessments of genetically engineered plants and food and feeds derived from genetically engineered plants.

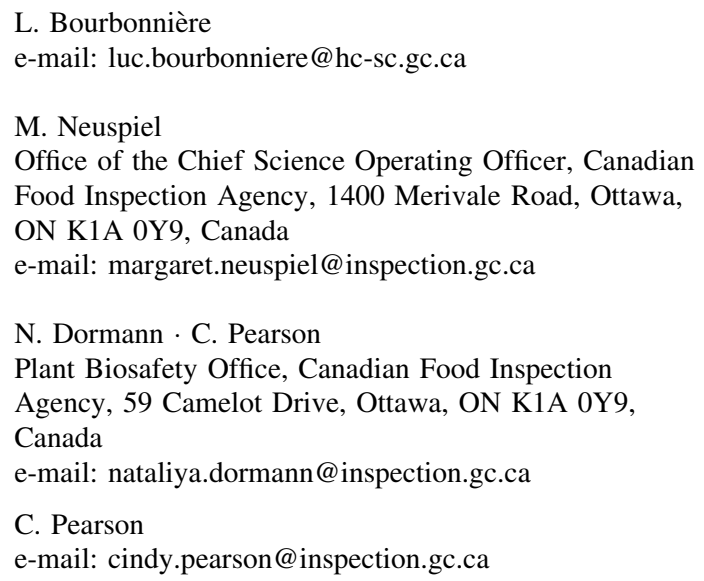

L. Bourbonnière

e-mail: luc.bourbonniere@hc-sc.gc.ca

M. Neuspiel

Office of the Chief Science Operating Officer, Canadian

Food Inspection Agency, 1400 Merivale Road, Ottawa,

ON K1A 0Y9, Canada

e-mail: margaret.neuspiel@inspection.gc.ca

N. Dormann · C. Pearson

Plant Biosafety Office, Canadian Food Inspection

Agency, 59 Camelot Drive, Ottawa, ON K1A 0Y9,

Canada

e-mail: nataliya.dormann@inspection.gc.ca

C. Pearson

e-mail: cindy.pearson@inspection.gc.ca 
Keywords Insertional effects - Novel trait . Unintended trait · Genetic engineering - Conventional breeding $\cdot$ Pre-market assessment

\section{Introduction}

Genetic engineering is frequently used to introduce new DNA sequences that contain one or more genes into plants, leading to the development of plants that exhibit novel traits. Given the complexity of plant cells and the current limitations of genetic engineering, unintended traits may result from genetic engineering in addition to the novel trait. Unintended traits are phenotypic changes in the plant that can materialize as a consequence of genetic changes such as DNA insertions, deletions and rearrangements, all of which can take place during genetic engineering, and the associated tissue culture process. These changes may include the introduction of a new plant characteristic, loss of a previously expressed plant characteristic or expression of a characteristic that is outside the range of what is typically observed in the plant species. The safety of novel traits intentionally introduced into plants during development is assessed during the premarket assessment of genetically engineered plants, however, unintended traits are by their nature unexpected, making it difficult to test for them directly.

In most countries, a comparative approach used in the pre-market assessment of genetically engineered (GE) plants and foods and feeds derived from GE plants (hereafter, GE plants, foods and feeds) provides a structure that enables the identification and evaluation of unintended traits. The comparative approach was initially developed for the pre-market assessment of genetically engineered organisms in 1986, and has been adopted and used by national regulatory bodies for over 15 years to conduct pre-market assessments on more than a hundred GE plants, foods and feeds, many of which have become integral parts of the agricultural production systems and food and feed chains of more than 20 countries (Codex Alimentarius Commission 2003; OECD 1986, 1992, 2003). The comparative approach is important because it recognizes that conventional plant varieties, foods and feeds have a history of safe use, and allows the pre-market assessment of the GE plant, food or feed to focus on differences between the GE plant, food or feed and a counterpart in order to determine potential safety concerns. The counterpart is typically the isogenic line closest to the GE plant with an established history of safe use, which is usually a conventional plant (i.e., not genetically engineered). This approach acknowledges the pre-existing nutritional profile, toxins, allergens and anti-nutrients of conventional plants, foods and feeds as well as the impact of the conventional plant and its associated agricultural practices on the environment.

Pre-market assessments, based on the comparative approach, function by defining the novel trait expressed in the GE product, identifying any unintended traits, and assessing the potential of these traits to adversely affect the environment, or impact the safety and/or nutritional quality of the food or the safety and/or efficacy of the feed derived from the GE plant. To accomplish this, pre-market assessments include a molecular characterization of the GE plant that considers the inserted DNA, expressed gene products and their impact on the GE plant, and a comparison of the composition and phenotype of the GE plant to that of a counterpart. The choice of which components and phenotypic characteristics to examine is, in part, determined by the nature of the novel trait, but it is generally broader to allow for the identification of unintended traits as well.

One source of unintended traits in GE plants is insertional effects, which are the changes to the transformed plant's genome that result from the process of inserting DNA by genetic engineering (Kessler et al. 1992; König et al. 2004). In addition to the intended DNA, in many cases additional DNA is also inserted. The additional DNA can originate from either the plant genome, the DNA being inserted or it can simply be filler DNA of unknown origin (Forsbach et al. 2003; Kim et al. 2003; Kohli et al. 1999; Krysan et al. 2002; Makarevitch et al. 2003; Sallaud et al. 2003; Windels et al. 2003). Also common are small deletions in the plant genomic DNA flanking the site of insertion. These deletions are typically less than $100 \mathrm{bp}$, although larger deletions are also occasionally observed (Forsbach et al. 2003; Kim et al. 2003; Krysan et al. 2002; Makarevitch et al. 2003). Large scale rearrangements may also occur during genetic engineering. Agrobacterium tumefaciens-mediated transformation and biolistics-mediated transformation are two commonly used approaches for creating genetically engineered plants. Both of these approaches can result in complex genetic inserts containing multiple copies and/or rearrangements of both the DNA intended to be inserted 
and the host plant DNA (Dan and Ow 2011; Stewart et al. 2011). For instance, in arabidopsis (Arabidopsis thaliana) transformed using A. tumefaciens, chromosomal translocations have been documented, where the flanking genomic DNA on either side of the inserted DNA mapped to two different chromosomes (Forsbach et al. 2003; Nacry et al. 1998). These insertional effects can alter patterns of gene expression in the plant or change the nature of RNA and/or proteins expressed by endogenous genes, either of which could result in an unintended trait in the plant.

The comparative approach to the pre-market assessment of GE plants, foods and feeds continues to be valid. However, there is now increased familiarity with genetic engineering techniques, as well as a growing body of scientific research both on the process of genetic engineering and on GE plants, foods and feeds themselves. In addition, advancements in molecular analysis techniques, particularly high-throughput sequencing and global profiling technologies, have given us an unprecedented understanding of the nature and the impact of genetic changes in plants. This raises the question of whether the comparative approach may be further refined.

It is, therefore, an opportune time to review and contextualize our understanding of insertional effects and their potential to result in unintended traits and to investigate how these insertional effects may be best evaluated within the current comparative approach to pre-market assessments. A simultaneous review of genetic changes that occur spontaneously as well as those that occur during conventional breeding will provide a useful comparison for insertional effects in keeping with the comparative approach to pre-market assessments. If insertional effects are found to be similar to other genetic changes that occur in plants, they should present a similar level of risk. It would then be reasonable to re-evaluate the information required for the comparative pre-market assessment of GE plants, foods and feeds.

\section{Scope}

This review will be limited to a discussion of potential unintended traits resulting from insertional effects. Other potential sources of unintended traits exist, including pleiotropic effects, which occur when a gene within the inserted DNA sequence confers multiple traits, and position effects, which are variations in the expression of genes within the inserted DNA that are dependent on the site of insertion.

\section{Insertional effects and unintended traits}

Although it is sometimes assumed that insertional effects are in and of themselves an indication that a GE plant will display an unintended trait, this is not an accurate conclusion. In a T-DNA insertional mutagenesis study in rice (Oryza sativa), examination of 22,665 field-grown T1 lines identified only 4,065 lines with a visible mutant trait (Chern et al. 2007). A study by El Ouakfaoui and Miki (2005) investigated the impact of the insertion of two marker genes on global gene expression profiles in four arabidopsis lines. In three independent experiments, reproducible changes in gene expression were not observed for any genes (with the exception of the introduced genes themselves) demonstrating that genes can be inserted without altering the global gene expression profile (El Ouakfaoui and Miki 2005).

Since not all insertional effects will result in an unintended trait, the question then becomes: what factors will determine whether an insertional effect will cause an unintended trait in a GE plant? In most cases, the DNA will need to be inserted within or near to an endogenous gene. The likelihood of this occurring will vary from species to species based on genome composition. For instance, gene rich regions are expected to account for $17 \%$ of the maize (Zea mays) genome with an average gene density of 1 gene per $40 \mathrm{~kb}$ (Barakat et al. 1997; Messing et al. 2004). In contrast, in rice, gene rich regions account for $24 \%$ of the genome and the average gene density is one gene every 4 to $5 \mathrm{~kb}$ (Barakat et al. 1997; Yu et al. 2002). Although some studies have suggested that transgene integration in $A$. tumefaciens-mediated transformation is biased towards actively transcribed genes, results of other studies indicate that at least some of this perceived insertion site bias is due to selection in insert identification (Francis and Spiker 2005; Kim and Gelvin 2007).

If the insertion occurs within or near to an endogenous gene or regulatory element, either the expression of associated endogenous gene(s) or the nature of the RNA(s) and/or protein(s) produced must then be affected in order for an insertional effect to result in an unintended trait. The expression of an 
endogenous gene can change if insertions, deletions and rearrangements within the gene render it nonfunctional, completely abolishing expression, or alter its pattern or level of expression. Regulatory elements within the inserted DNA can also influence the expression of nearby endogenous genes. This has been exploited in activation tagging, where DNA is inserted that contains a moderate to strong enhancer or promoter, such as the CaMV $35 \mathrm{~S}$ promoter, with the goal of transcriptionally activating nearby genes (Chern et al. 2007; Jeong et al. 2002). In one such study, it was found that the average distance between the inserted enhancer and the activated gene was $4.4 \mathrm{~kb}$, although this distance could be as great as $8 \mathrm{~kb}$ (Ichikawa et al. 2003). The second case, wherein the expressed sequence of the RNA(s) and/or protein(s) of an endogenous gene is changed, could occur by a number of mechanisms, for example due to insertions, deletions and/or rearrangements within the gene resulting in it being truncated, alternatively spliced, or if a novel chimeric gene is created.

Even if there are changes in the expression of an endogenous gene or the nature of the gene product that is expressed, these will not necessarily result in an unintended trait. The levels of expression of a given gene will vary based on genetic background and environment, so any changes within this range of expression may fall within normal variation and therefore not be biologically significant. For example, the expression of thousands of plant genes are frequently altered in response to stress (El Ouakfaoui and Miki 2005; Kawaura et al. 2006; Zheng et al. 2010). Even in the absence of stress, many genes are differentially expressed in plants grown at different locations and under different environmental conditions (Barros et al. 2010).

Furthermore, in cases where the function of the endogenous gene is compromised by insertional effects, it may still not result in an unintended trait because of the high level of gene redundancy in plant genomes. Genomic analysis of arabidopsis and rice has revealed gene redundancy throughout approximately one-third to one-half of the genome (Goff et al. 2002; The Arabidopsis Genome Initiative 2000; Yu et al. 2002). The buffering effect of duplicate genes is a legacy of polyploidization, which can be found in the ancestry of most plant species (Adams and Wendel 2005; Comai 2005). Duplicate copies of a damaged or aberrantly expressed gene can mask potentially detrimental phenotypes. For example, in 4,000 greenhouse-grown arabidopsis lines with insertions of a transposon in gene-coding regions, only 139 showed a visible mutant phenotype (Kuromori et al. 2006). In another example, only about $2 \%$ of a mutant soybean (Glycine max) population generated by fast neutron (FN) radiation showed an abnormal visual phenotype (Bolon et al. 2011).

Finally, changes to the nature of the RNA and/or protein that is expressed from an endogenous gene may also fail to result in an unintended trait due to the RNA and protein quality control systems active in plant cells. These quality control systems actively target aberrant RNA and protein for degradation (Buchberger et al. 2010; Doma and Parker 2007), minimizing their impact on the plant phenotype.

In conclusion, the relationship between genotype and phenotype in plants is complex, and the role of the environment cannot be ignored. In many ways, plants are buffered against the consequences of genomic changes by the high level of gene redundancy in plant genomes and by quality control systems active in plants. All of these factors influence whether or not an insertional effect will produce an unintended trait. Therefore, while insertional effects, i.e. the changes to the transformed plant's genome that result from the process of inserting DNA by genetic engineering, are unavoidable during genetic engineering, unintended traits are not always a consequence of these genetic changes. This conclusion is supported by the fact that detailed review of the nutritional composition of over 100 GE plants in the United States and Canada failed to identify adverse effects of genetic engineering on the composition of these plants (Price and Underhill 2013).

\section{Genetic changes similar to insertional effects occur in plants}

Plant genomes change over time in response to natural selection and genetic drift. Those spontaneous genetic changes that confer traits that better allow a plant to survive and adapt are maintained by the species. The genomes of crops have additionally been shaped by humans through conventional breeding, through the selection of desired traits that have arisen spontaneously or been created through mutagenesis. Despite the genetic changes associated with conventional 
breeding, there is a history of safe use of conventional crops. It is therefore useful to determine whether the genetic changes that occur in plants, either spontaneously or through conventional breeding, are similar to insertional effects as a consequence of genetic engineering.

The movement of transposable elements

The impact of the insertion of DNA through genetic engineering is probably most closely paralleled by the movement of transposable elements. Transposable elements can be found in the genomes of all plants. In arabidopsis, they account for at least $10 \%$ of the genome, in rice at least $24.9 \%$, and in maize at least $57 \%$ (Messing et al. 2004; The Arabidopsis Genome Initiative 2000; Yu et al. 2002). Typically ranging in size from hundreds to thousands of base pairs and, for autonomous elements, containing one or more open reading frames, the impact of their insertion into novel genomic loci is similar to that of the insertion of DNA via genetic engineering (Bennetzen 2000). In fact, many transposable elements were first identified by the mutant traits they generated and this phenomenon has been exploited in large scale insertional mutagenesis studies (Greco et al. 2001; Kuromori et al. 2006; Walbot 2000). Like DNA insertions via genetic engineering, if a transposable element inserts within or near a plant gene, it can inactivate the gene, alter its level or pattern of expression, or alter the nature of the RNA and/or protein that is expressed by that gene (Casacuberta and Santiago 2003). This can lead to the expression of novel traits. For instance, transposition of a doppia transposable element resulted in the formation of the R-r:std allele in maize, within which the $R$-s subcomplex is expressed in the aleurone layer of seeds, leading to its pigmentation (May and Dellaporta 1998; Walker et al. 1995). New cultivars of fruit have arisen from sports where the underlying genetic change is the insertion of a transposable element in close proximity to a plant gene. Research on grape (Vitis vinifera) berry colour has identified a connection between a lack of anthocyanin pigment (i.e. white berry) phenotype and the insertion of Gret1, a 10422 bp long retrotransposon, in the promoter region of $V v m y b A l$, a gene located in the berry colour locus that encodes a transcription factor that induces the anthocyanin pathway (Kobayashi et al. 2004). Insertion of this retrotransposon separates the
VvmybAl coding sequence from its promoter and impairs its transcription, thus preventing anthocyanin production. The opposite effect has been observed in blood oranges (Citrus sinensis), where the insertion of a Copia-like retrotransposon adjacent to the Ruby Myb gene gave rise to the induction of the anthocyanin biosynthesis pathway (Butelli et al. 2012).

As is the case with the insertion of DNA through genetic engineering, the movement of transposable elements is also associated with insertions, deletions and rearrangements. Although movement of transposable elements between plant species has been reported (El Baidouri et al. 2014), the movement of transposable elements within a genome is much more common and would make a greater contribution to genetic change. DNA transposons often leave behind a footprint at the site of their excision, which may include small deletions, inversions, and insertions, including sequence from the transposable element itself (Bennetzen 2000; Rinehart et al. 1997). Rearrangements can result from aberrant transposition, which is known to occur with DNA transposons such as the $A c / D s$ family (Gray 2000). As with the insertion of DNA through genetic engineering, these insertions, deletions and rearrangements that are associated with the movement of transposable elements can alter the nature and/or regulation of plant genes. In many cases, transposable element activities have played a significant part in plant evolution (Lisch 2013). For example, an in-depth analysis of transposable elements in sequenced crop genomes identified 51 transposable element-induced phenotypic changes associated with domestication or diversification of cultivated plants (Vitte et al. 2014).

The extent of movement of transposable elements in cultivated plants is not yet fully known. Most transposable elements are likely inactive, but some are known to be active in certain tissues or during certain developmental stages (Bennetzen 2000; Grandbastien 1998). Transposable elements may also become activated in response to stress, major genomic rearrangements, such as chromosome breaks, polyploidization, and hybridization, as well as during protoplast isolation, and cell and tissue culture (Bennetzen 2000; Grandbastien 1998; Parisod et al. 2010; Shan et al. 2005; Wang et al. 2010; Zou et al. 2011). In contrast to sporadic activation, some transposable elements, such as those identified in rice, appear to be continuously active under normal conditions of growth (Moon et al. 
2006; Naito et al. 2006). Estimates of the frequency of movement of transposable elements vary. For example, approximately one new insertion of the nDart transposable element was found in every four plants in a study of backcrosses with the rice cultivar Hoshinoyume (Fujino et al. 2005). In contrast, a single rice line was reported to accumulate dozens of de novo $m$ Ping transposable elements per generation (Naito et al. 2006).

Repair of double-strand breaks by nonhomologous end-joining

Double-strand breaks may be caused by physical stress on chromosomes, DNA replication across a nick, DNA crossing over during homologous recombination, or by reactive oxygen species, which are produced by the plant cell as a by-product of metabolism, in response to pathogen attack, or by exposure to environmental pollutants, ionizing radiation and ultraviolet light.

The main pathway for repair of double-strand breaks in plants is non-homologous end-joining (NHEJ). NHEJ is an error-prone recombination pathway, and deletions, insertions and rearrangements are often observed at the repair site where the two DNA ends have been rejoined (Gorbunova and Levy 1999). These are similar to the deletions, insertions and rearrangements that are often observed at the site of DNA insertion in plants having undergone genetic engineering. As with genetic engineering, if these deletions, insertions and rearrangements involve endogenous genes, the nature and/or regulation of these genes can be affected.

A hallmark of NHEJ repair sites is the presence of short repeats, sometimes referred to as microhomologies, that are typically $1-8$ bp in length (Gorbunova and Levy 1999; Morita et al. 2009). These will be present when the repair is mediated by annealing of the exposed ends at these repeats. Interestingly, microhomologies are also often observed at sites where DNA has been inserted by genetic engineering, between the inserted DNA and the flanking plant genome sequences (Gorbunova and Levy 1999; Somers and Makarevitch 2004). This is because both Agrobacterium-mediated and particle bombardment transformation methods rely on the plant's DNA repair pathways, in particular NHEJ, to introduce the DNA, although the exact mechanisms have not yet been fully elucidated (Makarevitch et al. 2003; Mayerhofer et al. 1991; Somers and Makarevitch 2004; Takano et al. 1997). Furthermore, the footprints created by excision of DNA transposons also contain these microhomologies because, once again, NHEJ is involved in repairing the double-strand break caused by excision (Huang and Dooner 2012; Rubin and Levy 1997). The common mechanism that underlies the generation of the insertions, deletions and rearrangements found at sites of DNA insertion via genetic engineering, double-strand break repair and DNA transposon excision is evidence that these insertional effects are not unique to genetic engineering.

The generation of double-strand breaks and their repair is also a key feature of conventional breeding. As the phylogenetic distance between a cultivated crop and its wild relative becomes wider, it becomes more difficult to create hybrids and introgress desired traits. In such cases, traits can be introgressed through chromosomal translocations. Infrequently, such translocations may occur spontaneously, but in many cases they must be induced, for instance by using ionizing radiation to cause double-strand breaks (Fedak 1999; Friebe et al. 1996). Ionizing radiation is also used to induce double-strand breaks in order to create mutations. The resulting mutants typically have deletions ranging in size from tens to millions of base pairs, as well as rearrangements, including inversions and chromosomal translocations (Cecchini et al. 1998; Li et al. 2001; Morita et al. 2009; Nambara et al. 1994; Sato et al. 2009; Schuermann et al. 2005; Shirley et al. 1992; Sun et al. 1992; Yan et al. 2007). Again, microhomologies are often observed at repair sites. A comparison of genetic changes found at sites of double-strand break repair resulting from genetic engineering, cleavage with a restriction endonuclease and mutagenesis with gamma irradiation can be found in Table 1, further highlighting their similarity.

Intracellular transfer of organelle DNA

The presence of non-coding sequences in plant nuclear genomes that are homologous to plastid and mitochondrial sequences (nuclear organelle DNAs) suggests that organelle DNAs have repeatedly been inserted into the nuclear genome. In some cases it is clear that organelle DNA was inserted intact in the nuclear genome while in other cases rearrangements are apparent. In rice and arabidopsis, approximately 
$25 \%$ of nuclear organelle DNAs occur within genes (Richly and Leister 2004), and in some cases sequences have become functional parts of coding regions (Noutsos et al. 2007). Once again, microhomologies found at the sites of insertion of nuclear organelle DNAs indicate that these sequences are inserted by NHEJ, and consistent with this mechanism, deletions and insertions were observed at sites of insertion (Huang et al. 2004). The insertion of organelle DNA into the nuclear genome is therefore similar to the insertion of DNA during genetic engineering. Like the insertion of DNA through genetic engineering, the nuclear organelle DNAs have the potential to alter the level or pattern of expression of endogenous genes or alter the nature of the RNA and/or protein expressed by endogenous genes.

The frequency with which organelle DNA is transferred to the nucleus is not well characterized, but there is evidence in tobacco (Nicotiana tabacum) that novel nuclear organelle DNAs are formed under typical conditions of growth. Huang et al. (2003) transformed the tobacco chloroplast genome with a nucleus-specific marker gene and found that the marker transferred to the nucleus at a rate of 1 in every 16,000 progeny. This number is presumed to be an underestimation of the true transfer rate, since it required transfer of the marker gene in a functional state. Wang et al. (2012) further demonstrated that the rate of transfer of DNA from the chloroplast to the nucleus increased up to tenfold in response to mild temperature stresses.

Other sources of genetic change in plants due to inserted DNA

There are additional possible sources of genetic change in plants that are similar to insertional effects. These changes have been previously reviewed (Bock 2010; Liu et al. 2012) and while it is not the intent of this publication to review all sources of genetic change in plants in detail, a few examples will be provided. Pararetroviruses are double stranded DNA viruses that infect plants. Although these viruses lack integrase enzymes, endogenous pararetrovirus DNA had been found in various plant genomes such as rice, tobacco, tomato (Solanum lycopersicum), potato (Solanum tuberosum), banana and plantain (Musa spp.), and petunia (Petunia spp.) (Liu et al. 2012; Staginnus et al. 2007; Staginnus and Richert-Pöggeler 2006). The 
mechanism by which these sequences are inserted into plant genomes has not been elucidated but is considered to likely involve NHEJ (Liu et al. 2012). Another example is processed pseudogenes. Pseudogenes are nonfunctional genomic sequences with significant sequence similarity to functional RNA or proteincoding genes (Vanin 1985). Processed pseudogenes, which are derived from retrotransposition events where double-stranded cDNAs derived from reverse transcription events are integrated into the genome (Zou et al. 2009), represent a source of inserted DNA in plants. Finally, A. tumefaciens and closely related bacteria are capable of transferring bacterial genes into plant genes, and this ability long predates genetic engineering (Gelvin 2000).

As we have illustrated here, the insertion of DNA via genetic engineering is similar to the movement of transposable elements and the transfer of organelle DNA and other DNA sequences to the nucleus; and the insertions, deletions and rearrangements that are often observed at the site of DNA insertion by genetic engineering are no different than those observed at sites where double-strand breaks have been repaired, which is reflective of their common underlying mechanism. This information demonstrates, therefore, that insertional effects are similar to other genetic changes that occur spontaneously in plants and during conventional breeding. By virtue of their association with the novel trait, insertional effects will be present throughout a variety as long as the novel trait is maintained. In contrast, spontaneous genetic changes will arise in a single plant and are less likely to become established in a variety. Similar to insertional effects, with conventional breeding many of the genetic changes will be closely linked to the desired trait that is being selected, and as a result these will be present throughout a variety as long as that desired trait is maintained. Therefore the strongest parallel can be drawn between the insertional effects of genetic engineering and the genetic changes associated with conventional breeding.

\section{Other genetic changes also occur in plants}

In the previous section, we considered those genetic changes that are most similar to insertional effects. However, it is also important to consider other types of genetic changes that occur both spontaneously and as a result of conventional breeding in plants, because it is within this background of genetic change that insertional effects occur. Although these other genetic changes may not be similar in nature to insertional effects, they have the potential to be similar in impact.

Spontaneous genetic change

Mutations occur spontaneously in all living organisms. For instance, in arabidopsis, spontaneous mutations occur at a rate of $7 \times 10^{-9}$, which translates into 1.75 mutations per generation per diploid plant (Ossowski et al. 2010; Weber et al. 2012). If this rate of genetic change were consistent in plants with larger genomes, the number of mutations per generation would be correspondingly larger. For example, based on the genome size of soybean (Schmutz et al. 2010), more than 10 mutations per generation would be expected to spontaneously occur. This level of mutation is consistent with reports that single-nucleotidepolymorphisms (SNPs) were detected every 48-2,000 base pairs in wheat (Triticum aestivum), soybean, and maize, with coding regions containing fewer SNPs than noncoding regions (Weber et al. 2012). These mutations may occur during DNA replication, either due to mispairing, which results in single base substitutions, or strand slippage, which results in small insertions and deletions. Spontaneous lesions can also form, where bases are either lost or modified, causing them to mispair and again leading to single base substitutions. We discussed already the ability of environmental pollutants, ionizing radiation and ultraviolet light to cause double-strand breaks. These can also alter the structure of DNA directly, leading to single base substitutions.

Spontaneous genetic changes can also occur during homologous recombination, which, like NHEJ, can repair double-strand breaks, but also introduces genetic variation during meiosis. During homologous recombination, copy number variants can result from misalignment between repeated sequences on homologous chromosomes, leading to unequal crossing over. In arabidopsis, copy number variants were found to occur at a rate of $1-3 \times 10^{-6}$ for a given gene cluster, suggesting that a copy number variant could be formed as often as 1 in every 700 seeds (Gaut et al. 2007; Jelesko et al. 2004). Intrachromosomal recombination can also occur, and the outcome can include sequence deletions, inversions, and gene conversion. 
Recombination between homologous sequences located at different genomic locations can lead to large chromosomal rearrangements, including translocations (Gaut et al. 2007).

In addition to the impacts of transposable elements on plant genomes already discussed, transposable elements can further alter plant genomes in ways for which there is no parallel observed during genetic engineering. For example, transposable elements will occasionally amplify genomic DNA sequences (Bennetzen 2000). This can lead to increases in gene copy number if the genomic DNA sequence contains a gene. Such amplifications can give rise to novel traits, as was observed for the tomato variety Sun1642, for which an elongated fruit trait has been attributed to the duplication of the gene $S U N$ during the movement of a retrotransposon (Xiao et al. 2008). In addition, the capture of genes and gene fragments by transposable elements can lead to the creation of new genes through exon shuffling (Bennetzen 2005). In rice, many of the Mutator-like DNA elements (MULEs) contain gene fragments, often from different chromosomal loci, and there is evidence that at least some of these gene fragments are being expressed (Jiang et al. 2004). Likewise in soybean, the Tgm-Express 1 transposon (of the CACTA super family) carries multiple captured gene fragments. The presence of this transposon in Intron 2 of the flavanone 3-hydroxylase gene $(F 3 H)$ (i.e. the $w p$ mutant flower colour gene) results in the generation of several chimeric transcripts from alternative splicing of the pre-mRNA (Zabala and Vodkin 2007). The expression of these transcripts (including a transcript identical to that of the wild type gene) most likely contributes to the resultant mutant (i.e. pink) flower phenotype.

Finally, on a larger scale, plants may sometimes loose or gain whole chromosomes. This occurs during cell division, when chromosomes are not distributed properly between cells. Similarly, mitotic or meiotic failures or the fusion of unreduced gametes can lead to whole genome duplications. There is evidence that most plants have undergone at least two, and as many as three to four, whole genome duplications at some point in their evolution, suggesting that this process has had a major impact on plant evolution (Adams and Wendel 2005; Jiao et al. 2011; Leitch and Leitch 2008). Whole genome duplications are typically followed by a series of rearrangements, including extensive sequence loss, homologous recombination, translocations, as well as adjustments to gene expression (Adams and Wendel 2005). Sorghum (Sorghum bicolour) and rice have largely collinear genomic maps with maize, permitting comparison of their chromosomal arrangements. A comparison of select genomic regions from these three species suggests that at least $50 \%$ of duplicate genes in maize were lost following polyploidization (Lai et al. 2004).

Clearly there are a number of mechanisms at work in plants that can bring about genetic changes. The cumulative impact of these genetic changes can be illustrated by comparative genomic analyses, which have demonstrated that plant genomes are remarkably diverse even within a given species. The maize genome, in particular, is known to be highly polymorphic. In a survey of the $3^{\prime}$ ends of 18 genes within 36 elite maize inbred lines, SNPs were found to occur on average every $60.8 \mathrm{bp}$ and small insertions and deletions every $126 \mathrm{bp}$, ranging in size from 1 to 400 bp (Ching et al. 2002). Larger scale rearrangements are also common between individuals within a species, with genes found in different locations, different copy numbers or even absent in some individuals and present in others (i.e. presence/ absence variation). A comparative genomic hybridization analysis of 19 maize genotypes and 14 teosinte genotypes using a custom microarray with 32,487 maize genes identified 1,065 genes with copy number variation and 2,824 genes that were present in some genotypes and absent in others (Swanson-Wagner et al. 2010). Similarly, a comparison of 2,094 contigs from the rice varieties Nipponbare and 93-11, which accounted for approximately $10 \%$ of the whole genome, revealed 156 genes that were asymmetrically located, 91 with copy number variation, and 82 genes that were present in one variety and absent in the other (Ding et al. 2007). Recently, the use of whole genome sequencing has revealed that the potato, maize, and soybean genomes possess dozens to thousands of presence/absence variations between individual varieties of their respective species (Lai et al. 2010; Lam et al. 2010; McHale et al. 2012; Potato Genome Sequencing Consortium 2011).

Furthermore, gene movement is extensive in large plant genomes such as wheat, barley (Hordeum vulgare), and their relatives. Comparisons between syntenic (i.e. collinear) regions of the wheat, Brachypodium, rice, and sorghum genomes show that the movement (or duplication) of syntenic genes to non- 
syntenic positions are most likely resultant of transposable element activity and double-strand break repair (Wicker et al. 2010). These mechanisms frequently result in the creation of a pseudogene that may or may not be functional in its new position in the genome. However, if the non-syntenic gene maintains the functional activity of its homolog (i.e. the gene from which it was duplicated), and the homolog is somehow lost, the non-syntenic gene may be selected for and maintained at its new position, further eroding collinearity between related plant species (Wicker et al. 2010, 2011).

Some types of genetic change, such as DNA replication errors, spontaneous lesions, and the formation of copy number variants during recombination, constantly occur in plants. In contrast, the amount of DNA damage from exposure to external mutagens is highly dependent on environmental conditions, and the activity of transposable elements will similarly be dependent on a number of internal and external factors, as already discussed. While large changes such as polyploidization and other ploidy changes occur infrequently, they do illustrate the remarkable ability of plants to undergo large-scale genomic changes. The frequency of polyploidization events in the evolutionary history of flowering plants suggests that large-scale genomic changes in some cases offer a selective advantage.

\section{Conventional breeding}

The most commonly applied conventional breeding technique is the introgression of a desired trait either from another individual within the same species, via intraspecific crossing, or from another species, typically a wild relative, via interspecific crossing. Genes for pest and disease resistance are more commonly targeted from these wild relatives. A study of 19 important commercial crops revealed evidence of introgression from 60 wild relatives into 13 of these crops (Hajjar and Hodgkin 2007). Another study on the use of introgression of disease resistance genes into wheat reported that six different genera have been used successfully as sources of these genes in wheat (Jones et al. 1995). Using these approaches, it is unlikely that only the gene responsible for the desired trait will be introduced into the final plant. Often, large amounts of DNA sequence surrounding the gene of interest will be transferred simultaneously, resulting in undesired traits being introduced along with the desired trait; this is known as linkage drag. Since some genes may be present in some individuals but not in others, intraspecific crosses have the potential to not only introduce novel alleles, but also novel genes. Linkage drag can be minimized by repeated backcrossing to eliminate more and more of the introgressed DNA sequence, but this process can be timeconsuming, and it may not be possible to completely eliminate all of the undesired traits. For instance, the $M i$ locus in tomato introgressed from $S$. peruvianum can only be reduced to $650 \mathrm{~kb}$ and contains a number of genes, including a transposase, several with similarity to the arabidopsis transport inhibitor response-1 (TIR-1), and one with similarity to an arabidopsis transcription factor (jumonji) family protein (Seah et al. 2007). In another study, a wide variation was observed in the sizes of introgressed regions surrounding the $T m-2$ gene of $S$. peruvianum, which was introgressed independently intro various tomato cultivars, with one cultivar containing an entire chromosome arm (Young and Tanksley 1989).

As the phylogenetic distance between a cultivated crop and its wild relative becomes wider, it becomes more difficult to create hybrids and introgress the desired traits. Techniques such as embryo rescue, somatic hybridization and chromosome doubling may be employed in order to first establish viable hybrids. As already discussed, ionizing radiation may be used to create double-strand breaks in order to induce chromosomal translocations. Hybridization between distantly related species and chromosome doubling can have genome-wide consequences, including complete or partial elimination of the chromosomes of one of the two parents, and genome rearrangements, including translocations and recombination (Du et al. 2008; Liu and Li 2007). The resulting hybrids are often asymmetric, with the majority of the genome originating from one parent with single chromosomes or chromosome fragments belonging to the other parent (Du et al. 2008; Faure et al. 2002; Liu and Li 2007; Tu et al. 2009; $\mathrm{Xu}$ et al. 2007). Three recombinant inbred lines (RILs) derived from the asymmetric hybridization between rice and its wild relative Zizania latifolia contained less than $0.1 \%$ of Z. latifolia DNA, and yet genomic variation (in the form of SNPs and small insertions and deletions) was observed at approximately $30 \%$ of the loci examined (Wang et al. 2005). In addition, hybridization can 
sometimes result in the activation of transposable elements, the activity of which can further alter the genome, as already discussed (Shan et al. 2005; Wang et al. 2010; Zou et al. 2011).

Mutagenesis is another conventional breeding technique that creates novel genetic variation from which desired traits can be selected. The FAO/IAEA Mutant Variety Database (mvgs.iaea.org/AboutMutantVarities.aspx) currently lists 3,218 mutant plant varieties that are released worldwide. In these varieties, mutations were most frequently induced by ionizing radiation (predominantly using gamma rays and X-rays), although chemical mutagens were occasionally used (Ahloowalia et al. 2004). As already discussed, ionizing radiation can induce double-strand breaks. These breaks are repaired by NHEJ and often lead to deletions and rearrangements, but it can also alter bases leading to single base substitutions. For instance, gamma ray-induced single base substitutions in the rice GA20ox-2 gene are responsible for the $s d 1$ mutation found in Japanese and American highyielding semi-dwarf rice varieties that are widely commercialized (Ashikari et al. 2002). The popularity of chemical mutagens, particularly ethyl methanesulfonate (EMS), has increased in recent years. Chemical mutagens predominantly induce single base substitutions (Cooper et al. 2008; Greene et al. 2003; Olsen et al. 1993; Talamè et al. 2008; Uauy et al. 2009; Xin et al. 2008). EMS alters guanine residues such that they pair with thymine as opposed to cytosine. Following DNA repair, the G/C pair may be converted to an A/T (Greene et al. 2003). Other alkylating agents such as dimethyl sulphate and diethyl sulphate are expected to have similar effects (Hoffmann 1980).

Finally, in both conventional breeding and genetic engineering, plants can be regenerated from cell or tissue culture, and this laboratory technique can give rise to genomic level changes known as somaclonal variation. Somaclonal variation is both an unintended effect of cell and tissue culture and a potential source of novel traits for selection. Somaclonal variation can take the form of single base substitutions, insertions, deletions, rearrangements or changes in chromosome number (Jiang et al. 2011; Karp and Maddock 1984; Lee and Phillips 1987; van den Bulk et al. 1990). In addition, transposable elements may be activated by cell and tissue culture. For instance, in regenerated rice plants, between 5 and 30 transposed copies of the retrotransposon Tos 17 could be detected, and the number of copies was found to increase with increased time spent in culture (Hirochika et al. 1996).

We have illustrated here that plant genomes are constantly changing in small ways, through errors in DNA replication or spontaneous lesions. They have also changed dramatically on a limited number of occasions in their evolutionary history through polyploidization. These genetic changes are necessary for their survival and adaptation and plants have a remarkable capacity to undergo major genetic change. In addition to these spontaneous genetic changes, plant genomes are further shaped by conventional breeding, which includes the introgression of foreign genes through breeding with wild relatives (Hajjar and Hodgkin 2007; Jones et al. 1995). Genomic comparisons have elegantly illustrated the cumulative impact of conventional breeding on plant genomes. This aspect has been exploited by plant breeders, creating novel varieties in conventional breeding, and recognized by seed production systems that include tolerance for off-types (http://seedgrowers.ca/).

For instance, a comparison of the genomes of 20 rice varieties and landraces determined that the modern indica and japonica varieties each had on average $8.4 \mathrm{Mb}$ of DNA introgressed from other varietal groups and the more traditional aus varieties had an average of $17.5 \mathrm{Mb}$ of introgressed DNA (McNally et al. 2009). In tomato, a study of sequence polymorphisms between fresh-market, processing, vintage and landrace varieties identified lower levels of variation in vintage and landrace varieties that was reflective of the reduced genetic diversity that developed during domestication as a result of genetic bottlenecks (Sim et al. 2009). Broader levels of variation were observed for the fresh-market and processing varieties, reflective of more recent tomato breeding practices in which new genetic variation is introgressed from wild species.

In conclusion, compared to genetic engineering, conventional breeding techniques can have a much larger impact on the plant genome. Hybridization, mutagenesis, and somaclonal variation all give rise to genome-wide genetic change. Similarly, introgression of a desired trait is frequently accompanied by additional unintended traits due to linkage drag, as well as introducing as many, if not more, genetic elements that could influence genes near to the site of introgression, compared to the insertion of DNA by genetic engineering. This premise is supported by 
several global gene expression profiling studies that illustrate that conventional breeding is more likely to impact the function of the genome than genetic engineering. For instance, mutagenesis of rice induced more changes in gene expression than the insertion of DNA by genetic engineering (Batista et al. 2008). Differences in gene expression are also typically greater between different conventionally bred cultivars than they are between a genetically engineered plant and an untransformed comparator, as demonstrated in wheat and soybean (Baudo et al. 2006; Cheng et al. 2008).

\section{Genetic changes and risk}

As has already been discussed, the relationship between genotype and phenotype is complex and it is also tempered by the environment. Genetic changes may be introduced into plants spontaneously or through conventional breeding or genetic engineering. The buffering capabilities of plant genomes and the quality control systems in plant cells will prevent many of these genetic changes from giving rise to discernible changes in a plant's phenotype. Even in those cases where genetic changes introduced during conventional breeding or genetic engineering do give rise to unintended traits, the unintended traits may not impact the safety of foods or feeds derived from the plant or the risk to the environment from cultivation of the plant. Any risks will depend on whether the unintended trait has an adverse impact on food or feed safety or the environment, such as production of a novel toxin or a change in weediness, and on how the event containing the unintended trait is developed and processed.

While it is theoretically possible that a new toxin, anti-nutrient or allergen might be introduced into a plant, the potential for this to occur is low. In fact, there are no documented cases where conventional breeding has resulted in the production of a novel toxin or allergen in a crop (Steiner et al. 2013). It is recognized that genetic changes from any type of crop development could alter levels of endogenous toxins or anti-nutrients in plants. However, in most commercially grown crops, these are well characterized and are often monitored to avoid development of crops with negative attributes.

Similarly, while traits that contribute to weediness can be introduced into crop varieties during the conventional breeding process, feral crop populations are rare (Warwick and Stewart 2005), suggesting that the potential for conventional breeding to make a crop weedy is low. This is particularly true for highly domesticated crops that are largely dependent upon cultivation for reproduction.

Another important consideration is how the use of plants-meaning their development, production, storage, processing and preparation-may play a role in managing the risks associated with genetic changes from either genetic engineering or conventional breeding. Cultivar development typically requires upwards of 10 years and involves the evaluation of thousands of plants, resulting in the selection of one or very few final cultivars. Throughout breeding and seed production, selection is applied to eliminate off-types, which are those plants that show an unintended trait. As mentioned above, specific tests may also be conducted on those plants that are known to produce toxins or anti-nutrients in order to eliminate lines that produce unacceptably high levels of such compounds. For instance, glycoalkaloid levels in potato tubers and glucosinolates in canola may be measured. Another important end goal of the selection process is uniformity, which helps to ensure that a cultivar performs consistently once commercially released. The end result of this selection process is that unintended traits which may have occurred are frequently eliminated.

The processing and preparation of foods and feeds may also play a role in managing the risks associated with genetic changes. For example, processing conditions that involve heat or pressure may significantly reduce the levels of toxins and/or anti-nutrients in the food or feed before consumption, so that any genetic changes that alter the levels of such compounds do not present a safety concern. As long as the plants continue to be used in the same way as their conventional counterparts, the risks will be managed to the same extent.

Within the realm of conventional breeding, unintended traits are evaluated primarily for their potential to improve a cultivar. The focus of development for most crops not developed by genetic engineering is on the introduction of desirable characteristics for producers, processors and end-users, without compromising pre-existing characteristics, and while maintaining uniformity. The safety of the genetic changes which occur as a result of conventional breeding is rarely considered, and restricted to known 
toxins and anti-nutrients. Despite this, examples of adverse effects on human or animal health or the environment are extremely rare, and when they have occurred, they have all involved known compounds, not novel ones (Steiner et al. 2013). There is a history of safe use in agriculture as well as food and feed of cultivars developed using conventional breeding. As a result of this history of safe use, in many countries premarket assessments are not conducted on new cultivars developed using conventional breeding.

\section{Conclusions}

A number of important conclusions may be drawn from this review of insertional effects and their potential to result in unintended traits. First, insertional effects are an unavoidable consequence of genetic engineering, but the introduction of unintended traits is not. Secondly, a comparison of insertional effects with other genetic changes that occur in plant genomes reveals that genetic changes similar to insertional effects occur spontaneously and during conventional breeding, for example by the movement of transposable elements, the transfer of organelle DNA to the nucleus and as a consequence of the repair of doublestrand breaks by NHEJ. A broader consideration of genetic changes that either occur spontaneously or are generated during conventional breeding illustrates that plant genomes are constantly changing (McClintock 1984; Weber et al. 2012), and therefore that insertional effects introduced through genetic engineering make a relatively small contribution to the final genetic makeup of given plant varieties. Finally, the impact of genetic changes in a plant, including but not limited to insertional effects, is influenced by the low potential that these genetic changes will result in a phenotypic change in the plant, and by the low potential for this phenotypic change to have an adverse effect on the plant, or the resulting food and feed safety.

It was hypothesized that if insertional effects are found to be similar to other genetic changes that occur in plants, they should present a similar level of risk. We have illustrated here that the insertional effects associated with genetic engineering are similar to the genetic changes that occur in conventionally bred plants. Based on this similarity, insertional effects should present a similar level of risk as genetic changes associated with conventional breeding. In light of this conclusion, it is reasonable to re-evaluate the information required for the pre-market assessment of genetically engineered plants, foods and feeds. In doing so, it will be important to maintain consistency with international guidelines, such as Codex Alimentarius (2003).

Although these conclusions are relevant to the consideration of all transformation events, a reevaluation of this information will be particularly useful for retransformants, which are plants that have been transformed with the identical construct(s) as a previously authorized plant of the same species that confers the same trait, and for which the safety of the trait has been previously assessed (CFIA 2012a, b). Retransformants are often generated for vegetatively propagated plants where it can be difficult to transfer a novel trait through sexual crosses, and therefore different cultivars are often independently transformed. Performing individual pre-market assessments on each transformant can be resourceintensive for both crop developers and regulatory authorities. If the genetic elements in the DNA that have been inserted in a retransformant are identical in sequence and order to those inserted in the original transformant, and genes are expressed at a similar level, the only potential difference between the original transformant that was assessed and a retransformant would be insertional effects. In such situations, the conclusions of this paper could be used to inform the pre-market assessment and determine appropriate information requirements.

Open Access This article is distributed under the terms of the Creative Commons Attribution License which permits any use, distribution, and reproduction in any medium, provided the original author(s) and the source are credited.

\section{References}

Adams KL, Wendel JF (2005) Polyploidy and genome evolution in plants. Curr Opin Plant Biol 8:135-141. doi:10.1016/j. pbi.2005.01.001

Ahloowalia BS, Maluszynski M, Nichterlein K (2004) Global impact of mutation-derived varieties Euphytica 135:187-204

Ashikari M et al (2002) Loss-of-function of a rice gibberellin biosynthetic gene, GA20 oxidase (GA20ox-2) led to the rice 'Green Revolution'. Breed Sci 52

Barakat A, Carels N, Bernardi G (1997) The distribution of genes in the genomes of Gramineae. Proc Natl Acad Sci USA 94:6857-6861 
Barros E, Lezar S, Anttonen MJ, van Dijk JP, Röhlig RM, Kok EJ, Engel K-H (2010) Comparison of two GM maize varieties with a near-isogenic non-GM variety using transcriptomics, proteomics and metabolomics. Plant Biotechnol J 8:436-451. doi:10.1111/j.1467-7652.2009.00487.x

Batista R, Saibo N, Lourenço T, Oliveira MM (2008) Microarray analyses reveal that plant mutagenesis may induce more transcriptomic changes than transgene insertion. Proc Natl Acad Sci USA 105:3640-3645. doi:10.1073/pnas. 0707881105

Baudo MM, Lyons R, Powers S, Pastori GM, Edwards KJ, Holdsworth MJ, Shewry PR (2006) Transgenesis has less impact on the transcriptome of wheat grain than conventional breeding. Plant Biotechnol J 4:369-380. doi:10. 1111/j.1467-7652.2006.00193.x

Bennetzen JL (2000) Transposable element contributions to plant gene and genome evolution. Plant Mol Biol 42:251-269. doi:10.1023/a:1006344508454

Bennetzen JL (2005) Transposable elements, gene creation and genome rearrangement in flowering plants. Curr Opin Genet Dev 15:621-627. doi:10.1016/j.gde.2005.09.010

Bock R (2010) The give-and-take of DNA: horizontal gene transfer in plants. Trends Plant Sci 15:11-22

Bolon Y-T et al (2011) Phenotypic and genomic analyses of a fast neutron mutant population resource in soybean. Plant Physiol 156:240-253

Buchberger A, Bukau B, Sommer T (2010) Protein quality control in the cytosol and the endoplasmic reticulum: brothers in arms. Mol Cell 40:238-252. doi:10.1016/j. molcel.2010.10.001

Butelli E et al (2012) Retrotransposons control fruit-specific, cold-dependent accumulation of anthocyanins in blood oranges. Plant Cell Online 24:1242-1255

Casacuberta JM, Santiago N (2003) Plant LTR-retrotransposons and MITEs: control of transposition and impact on the evolution of plant genes and genomes. Gene 311:1-11. doi:10.1016/S0378-1119(03)00557-2

Cecchini E, Mulligan BJ, Covey SN, Milner JJ (1998) Characterization of gamma irradiation-induced deletion mutations at a selectable locus in Arabidopsis. Mutat Res 401:199-206. doi:10.1016/s0027-5107(98)00009-8

CFIA (2012a) Directive 94-08 (Dir 94-08). Assessment criteria for determining environmental safety of plants with novel traits

CFIA (2012b) RG1 regulatory guidance: feed registration procedures and labelling standards

Cheng KC, Beaulieu J, Iquira E, Belzile FJ, Fortin MG, Strömvik MV (2008) Effect of transgenes on global gene expression in soybean is within the natural range of variation of conventional cultivars. J Agric Food Chem 56:3057-3067. doi:10.1021/jf073505i

Chern C-G et al (2007) A rice phenomics study-phenotype scoring and seed propagation of a T-DNA insertioninduced rice mutant population. Plant Mol Biol 65:427-438. doi:10.1007/s11103-007-9218-z

Ching A et al (2002) SNP frequency, haplotype structure and linkage disequilibrium in elite maize inbred lines. BMC Genet 3:19

Codex Alimentarius Commission (2003) Guideline for the conduct of food safety assessment of foods derived from recombinant-DNA plants. CAC/GL 45-2003
Comai L (2005) The advantages and disadvantages of being polyploid. Nat Rev Genet 6:836-846

Cooper JL et al (2008) TILLING to detect induced mutations in soybean. BMC Plant Biol 8:9

Dan Y, Ow DW (2011) Plant transformation technology revolution in last three decades: historical technology developments in plant transformation, vol 1. Bentham Science

Ding J, Araki H, Wang Q, Zhang P, Yang S, Chen J-Q, Tian D (2007) Highly asymmetric rice genomes BMC Genom $8: 154$

Doma MK, Parker R (2007) RNA quality control in eukaryotes. Cell 131:660-668. doi:10.1016/j.cell.2007.10.041

Du X-Z, Ge X-H, Zhao Z-G, Li Z-Y (2008) Chromosome elimination and fragment introgression and recombination producing intertribal partial hybrids from Brassica napus $\times$ Lesquerella fendleri crosses. Plant Cell Rep 27:261-271. doi:10.1007/s00299-007-0452-2

El Baidouri M et al (2014) Widespread and frequent horizontal transfers of transposable elements in plants. Genome Res 24:831-838

El Ouakfaoui S, Miki B (2005) The stability of the Arabidopsis transcriptome in transgenic plants expressing the marker genes nptII and uidA. Plant J 41:791-800. doi:10.1111/j. 1365-313X.2005.02350.x

Faure N, Serieys H, Bervillé A, Cazaux E, Kaan F (2002) Occurrence of partial hybrids in wide crosses between sunflower (Helianthus annuus) and perennial species $H$. mollis and H. orgyalis. Theor Appl Genet 104:652-660. doi: $10.1007 / \mathrm{s} 001220100746$

Fedak G (1999) Molecular aids for integration of alien chromatin through wide crosses. Genome 42:584-591. doi:10. 1139/g99-046

Forsbach A, Schubert D, Lechtenberg B, Gils M, Schmidt R (2003) A comprehensive characterization of single-copy T-DNA insertions in the Arabidopsis thaliana genome. Plant Mol Biol 52:161-176. doi:10.1023/a: 1023929630687

Francis KE, Spiker S (2005) Identification of Arabidopsis thaliana transformants without selection reveals a high occurrence of silenced T-DNA integrations. Plant $\mathrm{J}$ 41:464-477. doi:10.1111/j.1365-313X.2004.02312.x

Friebe B, Jiang J, Raupp WJ, McIntosh RA, Gill BS (1996) Characterization of wheat-alien translocations conferring resistance to diseases and pests: current status. Euphytica 91:59-87. doi:10.1007/bf00035277

Fujino K, Sekiguchi H, Kiguchi T (2005) Identification of an active transposon in intact rice plants. Mol Genet Genomics 273:150-157. doi:10.1007/s00438-0051131-z

Gaut BS, Wright SI, Rizzon C, Dvorak J, Anderson LK (2007) Recombination: an underappreciated factor in the evolution of plant genomes. Nat Rev Genet 8:77-84

Gelvin SB (2000) Agrobacterium and plant genes involved in T-DNA transfer and integration. Annu Rev Plant Biol 51:223-256

Goff SA et al (2002) A draft sequence of the rice genome (Oryza sativa L ssp japonica). Science 296:92-100. doi:10.1126/ science. 1068275

Gorbunova V, Levy AA (1999) How plants make ends meet: DNA double-strand break repair. Trends Plant Sci 4:263-269. doi:10.1016/s1360-1385(99)01430-2 
Grandbastien M-A (1998) Activation of plant retrotransposons under stress conditions. Trends Plant Sci 3:181-187. doi:10.1016/S1360-1385(98)01232-1

Gray YHM (2000) It takes two transposons to tango: transposable-element-mediated chromosomal rearrangements. Trends Genet 16:461-468. doi:10.1016/s01689525(00)02104-1

Greco R et al (2001) Transposon insertional mutagenesis in rice. Plant Physiol 125:1175-1177. doi:10.1104/pp.125.3.1175

Greene EA et al (2003) Spectrum of chemically induced mutations from a large-scale reverse-genetic screen in Arabidopsis. Genetics 164:731-740

Hajjar R, Hodgkin T (2007) The use of wild relatives in crop improvement: a survey of developments over the last 20 years. Euphytica 156:1-13

Hirochika H, Sugimoto K, Otsuki Y, Tsugawa H, Kanda M (1996) Retrotransposons of rice involved in mutations induced by tissue culture. Proc Natl Acad Sci USA 93:7783-7788

Hoffmann GR (1980) Genetic effects of dimethyl sulfate, diethyl sulfate, and related compounds. Mutat Res 75:63-129

Huang JT, Dooner HK (2012) The spectrum and frequency of self-inflicted and host gene mutations produced by the transposon Ac in maize. Plant Cell 24:4149-4162. doi:10. 1105/tpc.112.104265

Huang CY, Ayliffe MA, Timmis JN (2003) Direct measurement of the transfer rate of chloroplast DNA into the nucleus. Nature 422:72-76

Huang CY, Ayliffe MA, Timmis JN (2004) Simple and complex nuclear loci created by newly transferred chloroplast DNA in tobacco. Proc Natl Acad Sci USA 101:9710-9715. doi:10.1073/pnas.0400853101

Ichikawa $\mathrm{T}$ et al (2003) Sequence database of 1172 T-DNA insertion sites in Arabidopsis activation-tagging lines that showed phenotypes in T1 generation. Plant J 36:421-429. doi:10.1046/j.1365-313X.2003.01876.x

Jelesko JG, Carter K, Thompson W, Kinoshita Y, Gruissem W (2004) Meiotic recombination between paralogous RBCSB genes on sister chromatids of Arabidopsis thaliana. Genetics 166:947-957. doi:10.1534/genetics.166.2.947

Jeong D-H et al (2002) T-DNA insertional mutagenesis for activation tagging in rice. Plant Physiol 130:1636-1644. doi:10.1104/pp.014357

Jiang N, Bao Z, Zhang X, Eddy SR, Wessler SR (2004) PackMULE transposable elements mediate gene evolution in plants. Nature 431:569-573. http://www.nature.com/ nature/journal/v431/n7008/suppinfo/nature02953_S1.html

Jiang C et al (2011) Regenerant Arabidopsis lineages display a distinct genome-wide spectrum of mutations conferring variant phenotypes. Curr Biol 21:1385-1390. doi:10.1016/ j.cub.2011.07.002

Jiao Y et al (2011) Ancestral polyploidy in seed plants and angiosperms. Nature 473:97-100

Jones SS, Murray TD, Allan RE (1995) The development of disease resistance in wheat. Annu Rev Phytopathol 33:429-443

Karp A, Maddock SE (1984) Chromosome variation in wheat plants regenerated from cultured immature embryos. Theor Appl Genet 67:249-255. doi:10.1007/bf00317047

Kawaura K, Mochida K, Yamazaki Y, Ogihara Y (2006) Transcriptome analysis of salinity stress responses in common wheat using a 22 k oligo-DNA microarray. Funct Integr Genomics 6:132-142. doi:10.1007/s10142-0050010-3

Kessler D, Taylor M, Maryanski J, Flamm E, Kahl L (1992) The safety of foods developed by biotechnology. Science (New York, NY) 256:1747-1749 (1832)

Kim SI, Gelvin SB (2007) Genome-wide analysis of Agrobacterium T-DNA integration sites in the Arabidopsis genome generated under non-selective conditions. Plant $\mathrm{J}$ 51:779-791

Kim S-R, Lee J, Jun S-H, Park S, Kang H-G, Kwon S, An G (2003) Transgene structures in T-DNA-inserted rice plants. Plant Mol Biol 52:761-773. doi:10.1023/a:1025093101021

Kobayashi S, Goto-Yamamoto N, Hirochika H (2004) Retrotransposon-induced mutations in grape skin color Science 304:982

Kohli A, Griffiths S, Palacios N, Twyman RM, Vain P, Laurie DA, Christou P (1999) Molecular characterization of transforming plasmid rearrangements in transgenic rice reveals a recombination hotspot in the CaMV $35 \mathrm{~S}$ promoter and confirms the predominance of microhomology mediated recombination. Plant J 17:591-601. doi:10.1046/ j.1365-313X.1999.00399.x

König A et al (2004) Assessment of the safety of foods derived from genetically modified (GM) crops. Food Chem Toxicol 42:1047-1088

Krysan PJ, Young JC, Jester PJ, Monson S, Copenhaver G, Preuss D, Sussman MR (2002) Characterization of T-DNA insertion sites in Arabidopsis thaliana and the implications for saturation mutagenesis OMICS. J Integr Biol 6:163-174. doi:10.1089/153623102760092760

Kuromori T et al (2006) A trial of phenome analysis using $4000 \mathrm{Ds}$ insertional mutants in gene-coding regions of Arabidopsis. Plant J 47:640-651. doi:10.1111/j.1365-313X.2006.02808.x

Lai J et al (2004) Gene loss and movement in the maize genome. Genome Res 14:1924-1931. doi:10.1101/gr.2701104

Lai J et al (2010) Genome-wide patterns of genetic variation among elite maize inbred lines. Nat Genet 42:1027-1030

Lam H-M et al (2010) Resequencing of 31 wild and cultivated soybean genomes identifies patterns of genetic diversity and selection. Nat Genet 42:1053-1059

Lee M, Phillips RL (1987) Genomic rearrangements in maize induced by tissue culture. Genome 29:122-128. doi:10. $1139 / \mathrm{g} 87-021$

Leitch AR, Leitch IJ (2008) Genomic plasticity and the diversity of polyploid plants. Science 320:481-483. doi:10.1126/ science. 1153585

Li X et al (2001) A fast neutron deletion mutagenesis-based reverse genetics system for plants Plant J 27:235-242. doi:10.1046/j.1365-313x.2001.01084.x

Lisch D (2013) How important are transposons for plant evolution? Nat Rev Genet 14:49-61

Liu M, Li Z-Y (2007) Genome doubling and chromosome elimination with fragment recombination leading to the formation of Brassica rapa-type plants with genomic alterations in crosses with Orychophragmus violaceus. Genome 50:985-993. doi:10.1139/g07-071

Liu R, Koyanagi KO, Chen S, Kishima Y (2012) Evolutionary force of AT-rich repeats to trap genomic and episomal DNAs into the rice genome: lessons from endogenous pararetrovirus. Plant J 72:817-828 
Makarevitch I, Svitashev SK, Somers DA (2003) Complete sequence analysis of transgene loci from plants transformed via microprojectile bombardment. Plant Mol Biol 52:421-432. doi:10.1023/a:1023968920830

May BP, Dellaporta SL (1998) Transposon sequences drive tissue-specific expression of the maize regulatory gene $R$ $s$. Plant J 13:241-247. doi:10.1046/j.1365-313X.1998. 00010.x

Mayerhofer R et al (1991) T-DNA integration: a mode of illegitimate recombination in plants. EMBO J 10:697-704

McClintock B (1984) The significance of responses of the genome to challenge. Science 226:792-801. doi:10.1126/ science. 15739260

McHale LK et al (2012) Structural variants in the soybean genome localize to clusters of biotic stress-response genes. Plant Physiol 159:1295-1308

McNally KL et al (2009) Genomewide SNP variation reveals relationships among landraces and modern varieties of rice. Proc Natl Acad Sci USA 106:12273-12278. doi:10.1073/ pnas.0900992106

Messing J et al (2004) Sequence composition and genome organization of maize. Proc Natl Acad Sci USA 101:14349-14354. doi:10.1073/pnas.0406163101

Moon $\mathrm{S}$ et al (2006) Identification of active transposon $d T o k, \mathrm{a}$ member of the $h A T$ family, in rice. Plant Cell Physiol 47:1473-1483. doi:10.1093/pcp/pc1012

Morita R, Kusaba M, Iida S, Yamaguchi H, Nishio T, Nishimura M (2009) Molecular characterization of mutations induced by gamma irradiation in rice. Genes Genet Syst 84:361-370

Nacry P, Camilleri C, Courtial B, Caboche M, Bouchez D (1998) Major chromosomal rearrangements induced by T-DNA transformation in Arabidopsis. Genetics 149:641-650

Naito K et al (2006) Dramatic amplification of a rice transposable element during recent domestication. Proc Natl Acad Sci USA 103:17620-17625. doi:10.1073/pnas. 0605421103

Nambara E, Keith K, McCourt P, Naito S (1994) Isolation of an internal deletion mutant of the Arabidopsis thaliana ABI3 Gene. Plant Cell Physiol 35:509-513

Noutsos C, Kleine T, Armbruster U, DalCorso G, Leister D (2007) Nuclear insertions of organellar DNA can create novel patches of functional exon sequences. Trends Genet 23:597-601. doi:10.1016/j.tig.2007.08.016

OECD (1986) Recombinant-DNA safety considerations. Organisation for Economic Co-Operation and Development, Paris

OECD (1992) Safety considerations for biotechnology. Organisation for Economic Co-operation and Development, Paris

OECD (2003) Principles for the risk analysis of foods derived from modern biotechnology. Organisation for Economic Co-operation and Development, Paris

Olsen O, Wang X, von Wettstein D (1993) Sodium azide mutagenesis: preferential generation of A.T $\rightarrow$ G.C transitions in the barley Ant18 gene. Proc Natl Acad Sci USA 90:8043-8047

Ossowski S et al (2010) The rate and molecular spectrum of spontaneous mutations in Arabidopsis thaliana. Science 327:92-94. doi:10.1126/science.1180677

Parisod C et al (2010) Impact of transposable elements on the organization and function of allopolyploid genomes. New Phytol 186:37-45. doi:10.1111/j.1469-8137.2009.03096.x
Potato Genome Sequencing Consortium (2011) Genome sequence and analysis of the tuber crop potato. Nature 475:189-195

Price WD, Underhill L (2013) Application of laws, policies, and guidance from the united states and canada to the regulation of food and feed derived from genetically modified crops: interpretation of composition data. J Agric Food Chem 61:8349-8355

Richly E, Leister D (2004) NUPTs in sequenced eukaryotes and their genomic organization in relation to NUMTs. Mol Biol Evol 21:1972-1980. doi:10.1093/molbev/msh210

Rinehart TA, Dean C, Weil CF (1997) Comparative analysis of non-random DNA repair following $A c$ transposon excision in maize and Arabidopsis. Plant J 12:1419-1427. doi:10. 1046/j.1365-313x.1997.12061419.x

Rubin E, Levy AA (1997) Abortive gap repair: underlying mechanism for $D s$ element formation. Mol Cell Biol 17:6294-6302

Sallaud C et al (2003) Highly efficient production and characterization of T-DNA plants for rice (Oryza sativa L.) functional genomics. Theor Appl Genet 106:1396-1408. doi: $10.1007 / \mathrm{s} 00122-002-1184-\mathrm{x}$

Salomon S, Puchta H (1998) Capture of genomic and T-DNA sequences during double-strand break repair in somatic plant cells. EMBO J 17:6086-6095

Sato Y, Morita R, Katsuma S, Nishimura M, Tanaka A, Kusaba M (2009) Two short-chain dehydrogenase/reductases, NON-YELLOW COLORING 1 and NYC1-LIKE, are required for chlorophyll $b$ and light-harvesting complex II degradation during senescence in rice. Plant J 57:120-131. doi:10.1111/j.1365-313X.2008.03670.x

Schmutz J et al (2010) Genome sequence of the palaeopolyploid soybean. Nature 463:178-183. http://www.nature.com/ nature/journal/v463/n7278/suppinfo/nature08670_S1.html

Schuermann D, Molinier J, Fritsch O, Hohn B (2005) The dual nature of homologous recombination in plants. Trends Genet 21:172-181. doi:10.1016/j.tig.2005.01.002

Seah S, Telleen AC, Williamson VM (2007) Introgressed and endogenous $\mathrm{Mi}-1$ gene clusters in tomato differ by complex rearrangements in flanking sequences and show sequence exchange and diversifying selection among homologues. Theor Appl Genet 114:1289-1302. doi:10.1007/s00122-007-0519-z

Shan X et al (2005) Mobilization of the active MITE transposons mPing and Pong in rice by introgression from wild rice (Zizania latifolia Griseb.). Mol Biol Evol 22:976-990. doi:10.1093/molbev/msi082

Shirley BW, Hanley S, Goodman HM (1992) Effects of ionizing radiation on a plant genome: analysis of two Arabidopsis transparent testa mutations. Plant Cell 4:333-347

Sim S-C, Robbins MD, Chilcott C, Zhu T, Francis DM (2009) Oligonucleotide array discovery of polymorphisms in cultivated tomato (Solanum lycopersicum L.) reveals patterns of SNP variation associated with breeding. BMC Genom 10:466

Somers DA, Makarevitch I (2004) Transgene integration in plants: poking or patching holes in promiscuous genomes? Curr Opin Biotechnol 15:126-131. doi:10.1016/j.copbio. 2004.02.007

Staginnus C, Richert-Pöggeler KR (2006) Endogenous pararetroviruses: two-faced travelers in the plant genome. Trends Plant Sci 11:485-491 
Staginnus C et al (2007) Endogenous pararetroviral sequences in tomato (Solanum lycopersicum) and related species. BMC Plant Biol 7:24

Steiner H-Y et al (2013) Evaluating the potential for adverse interactions within genetically engineered breeding stacks. Plant Physiol 161:1587-1594. doi:10.1104/pp.112.209817

Stewart CN, Touraev A, Citovsky V, Tzfira T (2011) Plant transformation technologies. Wiley, New York

Swanson-Wagner RA, Eichten SR, Kumari S, Tiffin P, Stein JC, Ware D, Springer NM (2010) Pervasive gene content variation and copy number variation in maize and its undomesticated progenitor. Genome Res 20:1689-1699. doi:10.1101/gr.109165.110

Takano M, Egawa H, Ikeda J-E, Wakasa K (1997) The structures of integration sites in transgenic rice. Plant $\mathrm{J}$ 11:353-361. doi:10.1046/j.1365-313X.1997.11030353.x

Talamè V, Bovina R, Sanguineti MC, Tuberosa R, Lundqvist U, Salvi S (2008) TILLMore, a resource for the discovery of chemically induced mutants in barley. Plant Biotechnol J 6:477-485. doi:10.1111/j.1467-7652.2008.00341.x

The Arabidopsis Genome Initiative (2000) Analysis of the genome sequence of the flowering plant Arabidopsis thaliana. Nature 408:796-815. http://www.nature.com/ nature/journal/v408/n6814/suppinfo/408796a0_S1.html

Sun T-p, Goodman HM, Ausubel FM (1992) Cloning the Arabidopsis GA1 locus by genomic subtraction. Plant Cell 4:119-128. doi:10.1105/tpc.4.2.119

Tu Y, Sun J, Ge X, Li Z (2009) Chromosome elimination, addition and introgression in intertribal partial hybrids between Brassica rapa and Isatis indigotica. Ann Bot 103:1039-1048. doi:10.1093/aob/mcp045

Uauy C et al (2009) A modified TILLING approach to detect induced mutations in tetraploid and hexaploid wheat. BMC Plant Biol 9:115

van den Bulk RW, Löffler HJM, Lindhout WH, Koornneef M (1990) Somaclonal variation in tomato: effect of explant source and a comparison with chemical mutagenesis. Theor Appl Genet 80:817-825. doi:10.1007/bf00224199

Vanin EF (1985) Processed pseudogenes: characteristics and evolution. Annu Rev Genet 19:253-272

Vitte C, Fustier M-A, Alix K, Tenaillon MI (2014) The bright side of transposons in crop evolution. Brief Funct Genomics:elu002

Walbot V (2000) Saturation mutagenesis using maize transposons. Curr Opin Plant Biol 3:103-107. doi:10.1016/s13695266(99)00051-5

Walker EL, Robbins TP, Bureau TE, Kermicle J, Dellaporta SL (1995) Transposon-mediated chromosomal rearrangements and gene duplications in the formation of the maize $R-r$ complex. EMBO J 14:2350-2363

Wang Y-M, Dong Z-Y, Zhang Z-J, Lin X-Y, Shen Y, Zhou D, Liu B (2005) Extensive de novo genomic variation in rice induced by introgression from wild rice (Zizania latifolia Griseb.). Genetics 170:1945-1956. doi:10.1534/genetics. 105.040964

Wang N et al (2010) Transpositional reactivation of the Dart transposon family in rice lines derived from introgressive hybridization with Zizania latifolia. BMC Plant Biol 10:190

Wang D, Lloyd AH, Timmis JN (2012) Environmental stress increases the entry of cytoplasmic organellar DNA into the nucleus in plants. Proc Natl Acad Sci USA 109:2444-2448. doi:10.1073/pnas.1117890109

Warwick SI, Stewart CN (2005) Crops come from wild plantshow domestication, transgenes, and linkage together shape ferality. In: Gressel J (ed) Crop ferality and volunteerism. CRC Press, Boca Raton, pp 9-30. doi:10.1201/ 9781420037999.ch2

Weber N, Halpin C, Hannah LC, Jez JM, Kough J, Parrott W (2012) Crop genome plasticity and its relevance to food and feed safety of genetically engineered breeding stacks. Plant Physiol 160:1842-1853

Wicker T, Buchmann JP, Keller B (2010) Patching gaps in plant genomes results in gene movement and erosion of colinearity. Genome Res 20:1229-1237

Wicker T et al (2011) Frequent gene movement and pseudogene evolution is common to the large and complex genomes of wheat, barley, and their relatives. Plant Cell Online 23:1706-1718

Windels P, De Buck S, Van Bockstaele E, De Loose M, Depicker A (2003) T-DNA integration in Arabidopsis chromosomes. Presence and origin of filler DNA sequences. Plant Physiol 133:2061-2068. doi:10.1104/pp.103.027532

Xiao H, Jiang N, Schaffner E, Stockinger EJ, van der Knaap E (2008) A retrotransposon-mediated gene duplication underlies morphological variation of tomato fruit. Science 319:1527-1530. doi:10.1126/science. 1153040

Xin Z, Wang ML, Barkley NA, Burow G, Franks C, Pederson G, Burke J (2008) Applying genotyping (TILLING) and phenotyping analyses to elucidate gene function in a chemically induced sorghum mutant population. BMC Plant Biol 8:103

Xu C-Y, Wan-Yan R-H, Li Z-Y (2007) Origin of new Brassica types from a single intergeneric hybrid between $B$. rapa and Orychophragmus violaceus by rapid chromosome evolution and introgression. J Genet 86:249-257. doi:10. 1007/s12041-007-0032-9

Yan C, Yan S, Zeng X, Zhang Z, Gu M (2007) Fine mapping and isolation of $B c 7(t)$, allelic to OsCesA4. J Genet Genomics 34:1019-1027. doi:10.1016/s1673-8527(07)60115-5

Young N, Tanksley S (1989) RFLP analysis of the size of chromosomal segments retained around the Tm-2 locus of tomato during backcross breeding. Theor Appl Genet 77:353-359

Yu J et al (2002) A draft sequence of the rice genome (Oryza sativa L. ssp. indica). Science 296:79-92. doi:10.1126/ science. 1068037

Zabala G, Vodkin L (2007) Novel exon combinations generated by alternative splicing of gene fragments mobilized by a CACTA transposon in glycine max. BMC Plant Biol 7:38

Zheng J et al (2010) Genome-wide transcriptome analysis of two maize inbred lines under drought stress. Plant Mol Biol 72:407-421. doi:10.1007/s11103-009-9579-6

Zou C, Lehti-Shiu MD, Thibaud-Nissen F, Prakash T, Buell CR, Shiu S-H (2009) Evolutionary and expression signatures of pseudogenes in Arabidopsis and rice. Plant Physiol 151:3-15

Zou J et al (2011) De novo genetic variation associated with retrotransposon activation, genomic rearrangements and trait variation in a recombinant inbred line population of Brassica napus derived from interspecific hybridization with Brassica rapa. Plant J 68:212-224. doi:10.1111/j. 1365-313X.2011.04679.x 